\title{
Les Loranthaceae des zones guinéenne et soudano-guinéenne au Bénin et leurs hôtes
}

\author{
Janvier G. HOUÉNON ${ }^{1 *}$, Hounnankpon YÉDOMONHAN ${ }^{2}$, Aristide Cossi ADOMOU ${ }^{2}$, \\ Monique G. TOSSOU ${ }^{2}$, Madjidou OUMOROU ${ }^{1}$ et Akpovi AKOĖGNINOU ${ }^{2}$ \\ ${ }^{I}$ Département du Génie de l'Environnement, Ecole Polytechnique d'Abomey-Calavi, Université d'Abomey- \\ Calavi, Bénin. \\ ${ }^{2}$ Laboratoire de Botanique et d'Ecologie végétale, Faculté des Sciences et Techniques, Université d'Abomey- \\ Calavi, Bénin. \\ *Auteur correspondant, E-mail : janvier_houenon@yahoo.fr; Tél.: (00229) 66823330
}

\section{RÉSUMÉ}

Une prospection itinérante a été faite de 1996 à 2010 en zones guinéenne et soudano-guinéenne au Bénin en vue d'inventorier les différentes espèces de Loranthaceae et leurs hôtes. Les inventaires ont été réalisés le long de quelques routes et des sentiers dans les 45 communes des deux zones phytogéographiques. $\mathrm{Au}$ total, 4 genres (Agelanthus, Globimetula, Phragmanthera, Tapinanthus) et 10 espèces (Agelanthus brunneus, A. dodoneifolius, Globimetula braunii, G. cupulata, Phragmanthera capitata, P. nigritana, Tapinanthus bangwensis, T. belvisii, T. globiferus et $T$. sessilifolius). Parmi ces espèces, deux sont nouvelles pour la flore du Bénin, à savoir : Globimetula cupulata et Tapinanthus belvisii. La diversité taxonomique des hôtes est de 105 espèces réparties dans 85 genres, 33 familles et une seule classe qui est celle des Dicotylédones. Les familles les plus riches en espèces parasitées sont les Leguminosae (23,8\%), les Euphorbiaceae $(8,6 \%)$ et les Moraceae $(7,6 \%)$. Parmi les espèces hôtes, $36,2 \%$ sont des bois d'œuvre et de service, $49 \%$ sont médicinales et $20 \%$ sont à la fois médicinales et alimentaires.

(C) 2012 International Formulae Group. All rights reserved.

Mots clés: Loranthaceae, parasites, hôtes, Bénin, zones phytogéographiques.

\section{INTRODUCTION}

Les Loranthaceae sont communément appelées gui d'Afrique à cause de leurs similitudes morphologique et biologique avec le gui d'Europe (Viscum album L., Viscaceae). Presque toutes les espèces de cette famille sont des plantes hémiparasites épiphytoïdes sauf une espèce, Phrygilanthus aphyllus qui est un holoparasite et les espèces de trois genres Atkinsonia, Nuytsia et Gaïadendron, parasites épirhizoïdes (Hocking et Finéran, 1983 ; Edouard, 1989 ; Finéran et Hocking, 1983).

Les Loranthaceae sont présentes dans toutes les régions intertropicales et tempérées du monde: Afrique, Amérique, Asie, Australie, etc. (Barlow, 1983) avec une prédilection pour l'hémisphère Sud (Balle, 1982 ; Polhill et Wiens, 1998). Les auteurs ne s'accordent pas tous sur le nombre de ces parasites. C'est ainsi que pour Parker et 
Riches (1993), elles comportent 1000 espèces et 74 genres. Mais selon Polhill et Wiens (1998), elles sont 950 espèces réparties dans 77 genres. Contrairement aux Viscaceae d'Europe qui ont fait l'objet d'études exhaustives sur leur morphologie, leur cytologie et leur biologie, il y a très peu de documents écrits disponibles sur les Loranthaceae d'Afrique qui comportent pourtant 21 genres dont 19 sont endémiques au Continent (Polhill et Wiens, 1998). Les premiers documents qu'on peut lire sont les travaux de Mallamaire (1945), Chevalier (1946) et Ruysen (1957) qui signalaient la présence des espèces de Loranthaceae sur le karité d'Afrique. Mais récemment, en Afrique occidentale, des travaux scientifiques ont été entrepris au Ghana (Room, 1973), au Mali (Boudet et Lebrun, 1986), en Côte d'Ivoire (Traoré et al., 1995) et au Burkina-Faso (Boussim, 1991) pour répertorier et analyser quantitativement l'impact économique de ces plantes vasculaires hémiparasites.

Au Bénin, aucune étude spécifique sur ces plantes vasculaires parasites n'a été réalisée en notre connaissance pourtant, certaines d'entre elles occasionnent des dégâts considérables sur des essences ayant des intérêts économique et social (Boussim, 1991). Elles ont simplement fait depuis 1976 l'objet d'une collection botanique disponible à l'Herbier National du Bénin. D'après la Flore analytique du Bénin (Akoègninou et al., 2006), seulement 4 genres et 11 espèces ont été identifiés sur tout le territoire. Il paraît alors important de procéder à un inventaire qualitatif des espèces de cette famille et de leurs hôtes.

\section{MATÉRIEL ET MÉTHODES Milieu d'étude}

La zone d'étude est située entre les parallèles $6^{\circ} 20^{\prime}$ et $8^{\circ} 30^{\prime}$ de latitude Nord et les méridiens $1^{\circ} 35^{\prime}$ et $2^{\circ} 45^{\prime}$ de longitude Est. Sa superficie est de $30512 \mathrm{~km}^{2}$, soit $27,07 \%$ du territoire béninois (Figure 1). Sa population est estimée à 4613821 habitants, soit 68,33\% de la population pays (Anonyme, 2003). Le climat varie avec la latitude; ainsi du type subéquatorial dans la partie Sud du domaine avec 4 saisons, 2 saisons de pluie alternant avec 2 saisons sèches, il passe au type tropical humide de transition avec une seule saison de pluie et une saison sèche (Akoègninou, 2004). La pluviosité moyenne annuelle varie suivant deux gradients : un gradient ouest-est avec $972 \mathrm{~mm}$ de pluie à l'Ouest à Grand-Popo et $1376,1 \mathrm{~mm}$ à l'Est à Porto-Novo et un gradient sud-nord, avec 1332,1 mm à Cotonou au Sud et 1133,4 mm à Bantè au Nord. Les températures moyennes annuelles oscillent autour de $27^{\circ} 6$ au Sud à Cotonou et $28^{\circ}$ à Savalou au Nord de la zone. La couverture végétale est caractérisée par son extrême morcellement dû à des conditions climatiques, édaphiques et à l'action anthropique. Selon Akoègninou (2004), 3 domaines de végétation sont distingués : le domaine des formations littorales renfermant la mangrove, les forêts marécageuses et les fourrés littoraux; le domaine des forêts denses humides semidécidues où les formations sont en mosaïque avec les fourrés secondaires, les champs et les plantations de diverses espèces exotiques et enfin le domaine des forêts denses sèches, des forêts claires et des forêts galeries entremêlées de savanes et de champs.

\section{Méthode d étude}

L'inventaire floristique des Loranthaceae a été réalisé une fois tous les trois mois, d'août 1996 à avril 2003 pour prendre en compte les différentes phénophases des espèces. En effet, selon Loubreau-Callen et Damblon (1994), la période de floraison d'une plante varie dans le temps et l'identification des espèces des Loranthaceae est surtout basée sur la morphologie de la fleur. L'inventaire des parasites et de leurs hôtes a été réalisé le long de quelques routes et sentiers dans chacune des 45 communes des deux zones phytogéographiques du milieu d'étude (Figure 1). Le choix des sentiers et routes sillonnés a été opéré avec l'aide des agents spécialistes dans la protection des végétaux des Centres Régionaux pour la Promotion Agricole (CeRPA) qui ont une bonne connaissance des différentes formations 


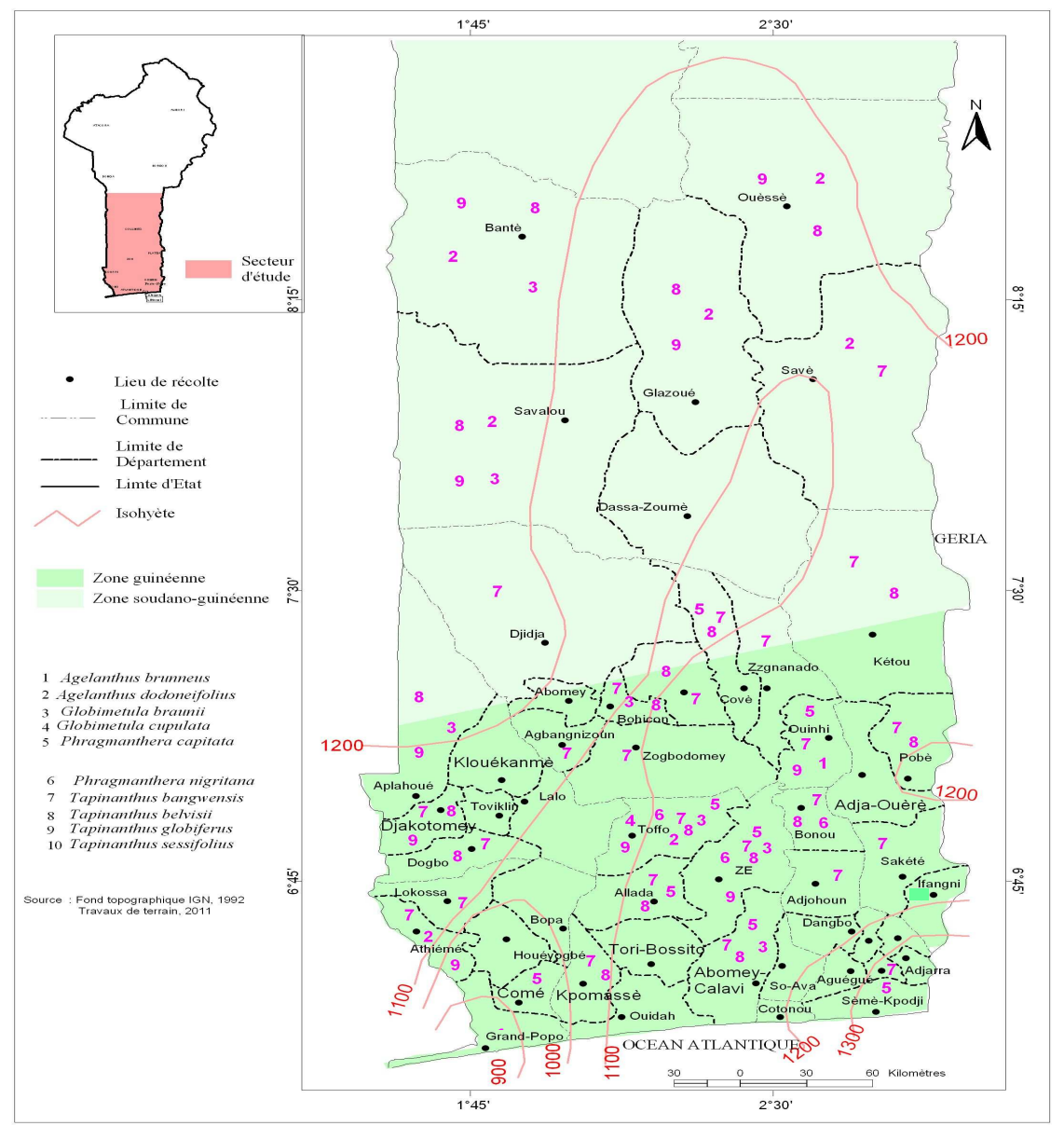

Figure 1: Localisation des sites prospectés et des espèces de Loranthaceae.

végétales et des vergers situés sur leur terroir. Tous les parasites rencontrés ont été systématiquement récoltés avec leurs hôtes. Les espèces fixées dans les cimes des grands arbres ont été observées aux jumelles et leurs hôtes ont été mentionnés. Les déterminations ont été faites à l'aide de la Flore de Hustchinson et Dalziel (1954-1972) et par comparaison avec les échantillons de Loranthaceae conservés au Centre National de Floristique (CNF) de l'Université de Cocody à Abidjan (Côte d'Ivoire). Elles ont été confirmées par le Professeur AKE ASSI Laurent dudit centre. La nomenclature adoptée a été celle de Akoègninou et al. (2006) et de Lebrun et Stork (1991-1997).
Les types biologiques considérés sont ceux de Aubreville (1963) à savoir : arbre = au moins $7 \mathrm{~m}$ de hauteur ; arbuste $=4$ à $7 \mathrm{~m}$ de hauteur ; suffrutex = peu lignifiée, moins de $4 \mathrm{~m}$ de hauteur.

En s'inspirant des travaux de Hoffmann (1994), un taux de spécificité parasitaire a été défini pour chaque parasite de même qu'une sensibilité parasitaire pour chaque hôte. La spécificité parasitaire est une caractéristique biologique essentielle chez les plantes dont l'existence dépend, en partie ou en totalité d'autres végétaux. Elle est obtenue en considérant le nombre d'hôtes affectés par le parasite sur le nombre total d'hôtes multiplié par cent. Elle est faible lorsque le parasite infeste de nombreuses espèces et forte 
si un petit nombre d'espèces sont parasitées. Ainsi des groupes de parasites ont été distingués : spécificité parasitaire très forte : [0 à 1\%], spécificité parasitaire forte: ]1 à 4\%], spécificité parasitaire moyenne: 14 à 9\%] inclus; spécificité parasitaire faible: > $9 \%$.

La sensibilité parasitaire des hôtes a été appréciée pour chaque plante hôte d'après le nombre d'espèces de parasites par hôte : peu sensible (1 à 2 parasites), sensible (3 à 4 parasites), fortement sensibles (5 à 6 parasites) et très fortement sensibles ( 7 parasites et plus).

\section{RÉSULTATS}

Diversité taxonomique des Loranthaceae

$\mathrm{Au}$ total, 10 espèces de Loranthaceae ont été recensées dans les zones guinéenne et soudano-guinéenne du Bénin (Tableau 1). Elles se répartissent dans 4 genres, à savoir : Agelanthus, Globimetula, Phragmanthera et Tapinanthus. Le genre Tapinanthus renferme 4 espèces, soit $40 \%$ de l'ensemble des espèces de parasites inventoriées. Il constitue ainsi le genre le plus riche en espèces. Les 3 autres genres comptent chacun 2 espèces.

En considérant la spécificité parasitaire, quatre classes de parasites sont distinguées (Tableau 1) La classe I des espèces à faible spécificité parasitaire, est plus diversifiée avec 6 espèces, soit $60 \%$ des Loranthaceae parasites. La classe II (spécificité parasitaire moyenne) réunit 2 espèces, soit $20 \%$. Les classes III (spécificité parasitaire forte) et IV (spécificité parasitaire très forte) ne renferment chacune qu'une seule espèce.

\footnotetext{
Répartition géographique des Loranthaceae

Des 10 espèces de Loranthaceae recensées, 6 (soit 60\%) sont communes aux zones guinéennes et soudano-guinéenne (Figure 1) et 4 (soit 40\%) ne sont rencontrées que dans la zone guinéenne. Aucune espèce n'est inventoriée exclusivement en zone soudano-guinéenne. La zone guinéenne abrite toutes les espèces de parasites et représente alors la zone la plus riche en Loranthaceae. Clés de détermination des Loranthaceae
}

\section{Clé des genres}

1- Loges d'anthères cloisonnées transversalement en plusieurs logettes superposées, plantes ayant au moins sur leurs organes végétatifs jeunes des poils étoilés ou à étage......3. Phragmanthera

1 '- Loges d'anthères non cloisonnées transversalement; plantes glabres ou presque, mais jamais portant des poils étoilés ou à étage.................................2

2- Tube de la corolle environ de même longueur que les lobes qui s'enroulent en spirale vers l'extérieur à l'anthèse ; stigmate plus ou moins conique et émarginé.... ...2.

\section{Globimetula}

2'- Tube de la corolle nettement plus long que les lobes qui restent dressés ou réfléchis à l'anthèse......................3

3 - Filets non prolongés par une dent apicale ; lobes de la corolle dressés............. 1. Agelanthus

3'- Filets prolongés par une dent apicale; lobes de la corolle réfléchis..................4. Tapinanthus

\section{Clé des espèces}

\section{Agelanthus Van Tiegh.}

1- Plante couverte de pustules rondes sur les rameaux donnant un aspect rugueux au toucher, de couleur gris noirâtre à l'état sec ; feuilles subsessiles, alternes ou parfois subopposées ; limbe obové, marqué de trois nervures saillantes partant de la base..........................1.1. A. brunneus 1'- Plante couverte de pustules fendillées perpendiculairement à l'axe des rameaux ; feuilles opposées ou verticillées, subsessiles ; limbe linéaire ou falciforme, portant quatre à six nervures latérales peu apparentes..............1.2. A. dodoneifolius

\section{Globimetula Van Tiegh.}

1- Feuilles généralement larges, elliptiques ou ovales, à base arrondie, obtuse ou cunéiforme ; limbe relativement mince....................2.1. G. braunii

1 '- Feuilles ovales à base arrondie ou cordée, roses ou rouges à l'état juvénile ; limbe épais. 2.1. G. cupulata

\section{Phragmanthera Van Tiegh.}


1- Lobes de la corolle dressés à l'anthèse, mesurant de 11 à $22 \mathrm{~mm}$ de longueur, peu élargis au sommet; anthères de 3 à $6,5 \mathrm{~mm}$ de long portant 6 à 10 logettes superposées verticalement; style non en "quille"........................3.1. P. capitata 1'- Lobes de la corolle réfléchis vers l'extérieur à l'anthèse, mesurant $9 \mathrm{~mm}$ environ de longueur, élargis en cuillère sur le $1 / 3$ supérieur de leur longueur ; anthères en rangs de 5 à 6 logettes superposées verticalement ; style en "quille".......................3.2. P. nigritana

\section{Tapinanthus Blume}

1-Feuilles pétiolées.........................2

1 '-Feuilles sessiles ou presque..........4.4.T. sessilifolius

2- Organes végétatifs jeunes et fleurs à poils simples ou portant des papilles; feuilles parfois alternes; renflement apical du bouton floral tronqué au sommet.........................2. T. belvisii

2'- Organes végétatifs entièrement glabres ; feuilles opposées ou subopposées ; renflement apical du bouton floral globuleux, ou ovoïde ou arrondi, mais jamais tronqué.. ...3

3- Renflement apical du bouton floral globuleux ou ovoïde.

4.1. $T$. bangwensis

3'- Renflement apical du bouton floral arrondi, surmonté de 5 gibbosités nettes, blanc crème ou rose et noircissant peu avant l'anthèse. 4.3. T. globiferus

Monographie des genres et espèces de Loranthaceae

Genre Agelanthus Van Tiegh.

Ce genre, comportant 59 espèces confinées à l'Afrique et à la péninsule arabique, est considéré comme le plus important en Afrique. Il affectionne 26 hôtes dans la zone d'étude. Agelanthus a un haustorium du type fusiforme BS-5 ou $\mathrm{CU}_{-2}$ quel que soit l'hôte avec un développement pas trop prononcé. De larges cordons diffus provoquent une hypertrophie de la partie infestée de l'hôte. Il est représenté par deux espèces dans la zone d'étude.

Agelanthus brunneus (Engl.) Balle et Hallé
Synonyme: Loranthus brunneus Engl., Tapinanthus brunneus (Engl.) Danser

Cette espèce est un arbrisseau d' $1 \mathrm{~m}$ au plus de hauteur; ses rameaux sont aplatis sur les parties jeunes. Les inflorescences sont à 1 , 2 ou 3 fleurs à l'aisselle des feuilles. Le tube de la corolle porte à son extrémité une coloration rose ou blanche. L'espèce est répandue depuis le Sénégal jusqu'en Angola. Elle est récoltée au Bénin dans la zone guinéenne à Houédja dans une forêt ripicole, le long du fleuve Ouémé. Elle parasite un seul hôte (Morelia senegalensis). Elle est dotée d'une très forte spécificité parasitaire égale à 0,9\% et fleurit tout au long de l'année.

Agelanthus dodoneifolius (DC.) Polhill et Wiens

Synonyme: Loranthus dodoneifolius DC., Scurrula dodoneifolia G. Don.

C'est un arbuste à rameaux généralement pendants mesurant jusqu'à $2 \mathrm{~m}$ de longueur. Les feuilles sont de couleur vertglauque. La corolle forme un long tube sans renflement apical avec des lobes dressés à l'anthèse. A. dodoneifolius est répandue depuis le Sénégal en Uganda. Au Bénin, elle est fréquemment rencontrée en zone soudanoguinéenne mais quelques fois en zone guinéenne, où elle parasite exclusivement Ceiba pentendra au Sud-Ouest du pays où la pluviométrie annuelle est la plus faible (900 à $1000 \mathrm{~mm}$ d'eau). Cette espèce affectionne 25 hôtes. Elle est de spécificité parasitaire faible $(23,8 \%)$. La floraison débute en juillet et les premiers fruits sont observés en octobre.

\section{Genre Globimetula Van Tiegh.}

Le genre Globimetula compte 13 espèces réparties dans toute l'Afrique intertropicale (Balle et Hallé, 1962 ; Polhill et Wiens, 1998). L'haustorium observé chez Globimetula est du type $\mathrm{CU}_{-1}$, quel que soit l'hôte ; ici c'est une union amplexicaule qui provient de la prolifération des tissus du parasite à la base qui cherche à embrasser la tige de l'hôte sur la face inférieure.

\section{Globimetula braunii (Engl.) Danser}

Synonyme: Loranthus Braunii Engl., Globimetula unguiformis (Engl.) Tiegh.

C'est une plante à rameaux pendants ou érigés et dont les feuilles sont épaisses et de 
taille variable. Le suçoir est en pince qui tend à encercler le rameau parasité. L'inflorescence est une ombelle de 4 à 8 fleurs souvent groupées aux vieux nœuds; le renflement apical du bouton floral est légèrement ailé. $G$. braunii est une espèce des forêts guinéocongolaises qui va de la Côte d'Ivoire au Sud du Soudan et à l'Ouest du Kenya. Au Bénin, l'espèce est récoltée sur 43 hôtes réparties dans les deux zones phytogéographiques. Elle est dotée d'une spécificité parasitaire faible (41\%) et est en fleur aux mois de Mai, Juin, Juillet, Septembre et Novembre.

Globimetula cupulata (DC.) Danser

Synonyme : Loranthus cupulatus DC.

La plante est longuement lianescente avec des rameaux abondamment ramifiés qui peuvent recouvrir entièrement l'hôte. Les jeunes feuilles, de couleur rougeâtre, permettent de reconnaître l'espèce à distance. Les rameaux peuvent s'interparasiter. L'espèce est répandue sur la côte ouestafricaine et dans les régions basses allant du Sud Sénégal en Sierra Léone. Au Bénin, elle est observée uniquement en zone guinéenne. Elle est rencontrée sur 6 hôtes et se parasite elle même. Elle est dotée d'une spécificité parasitaire moyenne $(5,7 \%)$. Sa floraison a lieu en avril, mai, juin, novembre et décembre; la fructification intervient en juillet, août, septembre.

\section{Genre Phragmanthera Van Tiegh.}

$\mathrm{Ce}$ genre renferme 34 espèces largement réparties en Afrique tropicale et en Arabie. Certaines espèces deviennent de véritables pestes dans les plantations. Le genre Phragmanthera présente l'une ou l'autre des formes d'haustorium suivantes: $\mathrm{BS}_{-1}, \mathrm{CU}_{-1}$ ou $\mathrm{CU}_{-3}$ selon l'espèce parasite. L'haustorium qui se présente sous forme d'un cordon massif, se ramifie à l'intérieur de l'hôte et finit par être visible à l'extérieur ; ce qui donne naissance à des rejets. Nous avons observé cette image sur Anacardium occidentale infestée par $P$. capitata dans la localité de Sèmè. La forme CU-1 de $P$. nigritata n'est pas aussi prononcée sur Citrus observée à Toffo.
Le genre est représenté au Bénin par deux espèces parasites de 32 hôtes.

Phragmanthera capitata (Sprengel) Ball.

Synonyme : Exostemma capitata Sprengel, Loranthus capitatus (Sprengel) Engl.

Tous les organes, végétatifs ou reproducteurs, de la plante portent des poils simples et des poils étoilés ou à étages, rapidement caducs avec l'âge. L'inflorescence est en ombelles de fleurs sessiles ou subsessiles brillamment colorées en jaunedoré, ce qui permet de reconnaître la plante de loin pendant la floraison. L'espèce est largement répandue en Afrique depuis la Guinée jusqu'au Congo démocratique et en Angola. Au Bénin, elle est en zones guinéenne et soudano-guinéenne. Elle affectionne 26 hôtes, plus particulièrement les plantes cultivées comme : Citrus reticulata, Leucaena leucocephala, Cola nitida, Citrus sinensis. Sa spécificité parasitaire est faible (24,8\%). L'espèce est en fleurs et/ou fruits de février à novembre.

\section{Phragmanthera nigritana (Hook.f. ex. Benth.) Balle}

Synonyme : Loranthus nigritanus Hook.f. ex Benth., Tapinanthus nigritanus (Hook.f. ex Benth.) Danser

Les organes végétatifs jeunes de la plante, les fleurs et les fruits sont recouverts de poils roux, dendritiques à étage et ou simples. L'inflorescence est en ombelles fasciculées. L'espèce est répandue dans les forêts galeries et les formations adjacentes depuis le Sud-Est du Ghana jusqu'au Cameroun et en Angola. Au Bénin, le parasite est rencontré dans la zone guinéenne. Il affectionne 6 hôtes, plus particulièrement Citrus spp. Il est de spécificité parasitaire moyenne $(5,7 \%)$. Il est en fleurs et/ou fruits aux mois d'avril, mai, juillet et septembre.

\section{Genre Tapinanthus Blume}

Avec plus de 200 espèces réparties dans toute l'Afrique à l'exception du Sahara occidental et oriental, Tapinanthus est le genre des Loranthaceae le plus représenté sur le continent. Il vit en parasite sur 98 hôtes autochtones ou exotiques. 
Le genre Tapinanthus présente la forme d'haustorium unique du type WR-1 quelle que soit l'espèce et la nature de l'hôte. Cette forme provient d'une prolifération des tissus de l'hôte autour de la base du parasite. Le genre comporte 4 espèces dans la zone d'étude.

\section{Tapinanthus bangwensis (Engl. \& Krause)} Danser

Synonyme: Loranthus bangwensis Engl. et K. Krause, Tapinanthus globiferus subsp. bangwensis (Engl. et K. Krause) Balle.

Tapinanthus bangwensis est une espèce à rameaux glabres retombants, fixée à son hôte par un haustorium volumineux. Le fruit est une baie rouge, ovoïde-globuleux. En Afrique de l'Ouest, l'espèce est rencontrée depuis le Sénégal jusqu'au Cameroun. Au Bénin, elle est observée surtout dans la zone guinéenne, mais quelquefois dans la zone soudano-guinéenne. Elle est parasite de 33 espèces aussi bien spontanées (Ficus sur, Vitex doniana) que cultivées (Artocarpus altilis, Citrus sinensis, Irvingia gabonensis, etc.). Sa spécificité parasitaire est alors faible $(31,3 \%)$. L'espèce est en fleurs et fruits presque tout au long de l'année.

\section{Tapinanthus belvisii (DC.) Danser}

Synonyme: Loranthus belvisii DC., Loranthus lanceolatus P. Beauv.

C'est une espèce à organes végétatifs jeunes pubescents. La tige principale est très courte et mesure 1 à $1,5 \mathrm{~cm}$ avant les premières bifurcations. Son haustorium est en forme de cône renversé. Les inflorescences sont en ombelles de 4 à 10 fleurs. T. belvisii est répandue en Afrique depuis la Guinée jusqu'au Congo et en Angola. Au Bénin, l'espèce affectionne 19 hôtes répandus dans toute la zone d'étude. Elle est particulièrement rencontrée dans les plantations d'agrumes. $\mathrm{Sa}$ spécificité parasitaire est faible $(18,1 \%)$. Elle fleurit tout au long de l'année sauf en mai et juin.

Tapinanthus globiferus (A. Rich.) Van Tieghen

Synonyme: Loranthus globiferus A. Rich., Loranthus verrucosus Engl.

La tige principale de $T$. globiferus est toujours courte et se ramifie abondamment dans tous les sens; les feuilles sont de taille très variables. L'inflorescence est en ombelles axillaires souvent réunies en fascicules. La tête renflée du bouton floral est diversement colorée; à l'anthèse, les lobes de la corolle sont réfléchis en arrière tandis que les filets des étamines sont recroquevillés, seul le style en quille reste dressé. En Afrique, l'espèce est rencontrée depuis la Guinée jusqu'au Nigéria. Elle est observée dans tout le domaine d'étude. T. globiferus affectionne 43 espèces cultivées ou non dont Agelanthus dodoneifolius et plus particulièrement Citrus spp. Elle est dotée d'une spécificité parasitaire faible $(41 \%)$ et est en fleur tout au long de l'année.

\section{Tapinanthus sessilifolius (P. Beauv.) Van Tieghen}

Synonyme : Loranthus sessilifolius $\mathrm{P}$. Beauv., Loranthus warneckei Engl.

La plante est pubescente avec des rameaux de $1 \mathrm{~m}$ de longueur, aplatis ou élargis aux nœuds près de l'extrémité. Les feuilles sont opposées ou subopposées, ovées ou ovélancéolées et cordées à la base. L'inflorescence est en ombelles de 2 à 12 fleurs sessiles ou presque. L'espèce est rencontrée depuis le Togo jusqu'au Nigéria. $\mathrm{Au}$ Bénin, T. sessilifolius est récoltée uniquement en zone guinéenne à Djondji sur Citrus aurantifolia, Newbouldia laevis et Laguncularia racemosa. L'espèce est de spécificité parasitaire forte $(2,9 \%)$. Elle est en fleur en février et mars.

\section{Diversité spécifique des hôtes des Loranthaceae}

$\mathrm{Au}$ total, 105 espèces-hôtes ligneuses sont recensées (Tableau 2). Elles sont réparties en 85 genres et 33 familles appartenant toutes à la classe des Dicotylédones.

Ficus est le genre le plus diversifié avec 5 espèces, soit 5,9\% des plantes-hôtes. Il est suivi du genre Albizia (4 espèces, soit $4,7 \%$ ) puis des genres Citrus, Cola et Terminalia qui renferment chacun 3 espèces, soit 3,5\%. Sept autres genres comptent chacun 2 espèces (2,4\%), à savoir : Acacia, Erythrina, Gardenia, Jatropha, Lannea, Pterocarpus et 
Senna. Ces 12 genres totalisent $37,7 \%$ de la richesse spécifique des plantes-hôtes. Les 73 genres restants sont tous monospécifiques.

Les Leguminosae constituent la famille la plus représentée avec 25 espèces, soit $23,8 \%$ dont $10,5 \%$ de LeguminosaeMimosoideae, 7,6\% de LeguminosaeCaesalpinioideae et $5,7 \%$ de LeguminosaePapilionoideae. Viennent ensuite, les Euphorbiaceae avec 9 espèces $(8,6 \%)$, les Moraceae avec 8 espèces (soit 7,6\%), les Sterculiaceae, les Combretaceae et les Rubiaceae avec 6 espèces chacune (soit $5,7 \%$ ). Les familles restantes ont chacune entre $4(3,8 \%)$ et 1 espèce $(0,9 \%)$.

Sur le plan morphologique, les plantes hôtes se répartissent dans trois groupes, à savoir :

- les arbres qui comptent 78 espèces, soit $74,3 \%$;

- les arbustes qui renferment 23 espèces, soit $21,9 \%$;

- les suffrutex qui comprennent 4 espèces recensées, soit 3,8\%.

Parmi les espèces hôtes, 2 (soit 1,9\%) sont elles-mêmes des Loranthaceae parasites. Il s'agit de Agelanthus dodoneifolius et de Globimetula cupulata. Ces deux espèces hôtes peuvent être alors appelées des hôtesparasites. Les 103 espèces restantes (soit $98,1 \%$ ) sont des hôtes non parasites.

En considérant l'utilité des planteshôtes, elles se regroupent en 3 classes. La première, celle des bois d'œuvre et de service, renferme 38 espèces, soit $36,2 \%$ des plantes hôtes. La deuxième est celle des plantes médicinales où l'on dénombre 52 espèces $(49,5 \%)$. La dernière, celle des plantes alimentaires, est riche de 21 espèces (20\%).

Parmi les plantes-hôtes, 86 espèces sont autochtones, soit $81,9 \%$ et 19 sont exotiques, soit $18,12 \%$.

Quatre classes de plantes hôtes sont distinguées en fonction de leur sensibilité parasitaire.

Parmi les 105 espèces-hôtes identifiées, 79 (soit 75,2\%) sont infestées par 1 ou 2 espèces et constituent la classe I (peu sensible). On peut citer par exemple Caloptropis procera, Jatropha multifida, Khaya senegalensis, Persea americana, Triplochiton scleroxylon et Vitex doniana.

La classe II des hôtes sensibles comprend 20 espèces (soit 19,1\%) dont Adansonia digitata, Ceiba pentandra, Irvingia gabonensis, Morinda lucida, Newbouldia laevis, Parkia biglobosa, etc.

La classe III (fortement sensible) est riche de 4 espèces, soit 3,8\%, à savoir : Acacia auriculiformis, Citus reticulata, Senna siamea et Tectona grandis.

La classe IV contient seulement Citrus sinensis qui est la seule plante-hôte ayant une très forte sensibilité parasitaire.

Tableau 1: Diversité taxonomique des Loranthaceae.

\begin{tabular}{lll}
\hline Espèces & ZC & SP (\%) \\
\hline Agelanthus brunneus (Engl.) Balle \& Hallé & $\mathrm{G}$ & $0,95^{* * * *}$ \\
Agelanthus dodoneifolius (DC.) Polh. \& Wiens & $\mathrm{G}-\mathrm{SG}$ & $23,80^{*}$ \\
Globimetula braunii (Engl.) Danser & $\mathrm{G}-\mathrm{SG}$ & $40,95^{*}$ \\
Globimetula cupulata (DC.) Danser & $\mathrm{G}$ & $5,71^{* *}$ \\
Phragmanthera capitata (Spreng.) Balle & $\mathrm{G}-\mathrm{SG}$ & $24,75^{*}$ \\
Phragmanthera nigritana (Hook.f. ex Benth.) Balle & $\mathrm{G}$ & $5,71^{* *}$ \\
Tapinanthus bangwensis (Engl. \& Krause) Danser & $\mathrm{G}-\mathrm{SG}$ & $31,43^{*}$ \\
Tapinanthus belvisii (DC.) Danser & $\mathrm{G}-\mathrm{SG}$ & $18,09^{*}$ \\
Tapinanthus globiferus (A. Rich.) Tiegh. & $\mathrm{G}-\mathrm{SG}$ & $40,95^{*}$ \\
Tapinanthus sessilifolius (P. Beauv.) Tiegh. & $\mathrm{G}$ & $2,86^{* * *}$ \\
\hline
\end{tabular}

$\mathrm{ZC}$ : zone phytogéographique de présence du parasite (G: zone guinéenne, SG : zone soudano-guinéenne) ; SP : spécificité parasitaire $(*$ : faible, $* *$ : moyenne, $* * *$ : forte, $* * * *$ : très forte). 
J G. HOUÉNON et al. / Int. J. Biol. Chem. Sci. 6(4): 1669-1686, 2012

Tableau 2: Liste des espèces hôtes avec leur sensibilité face aux espèces parasites.

\begin{tabular}{|c|c|c|c|c|c|c|c|c|c|c|c|c|c|}
\hline \multirow{2}{*}{ Hôtes } & \multirow{2}{*}{ TM } & \multicolumn{10}{|c|}{ Espèces de parasite } & \multirow{2}{*}{ Np } & \multirow{2}{*}{$\mathbf{S p}$} \\
\hline & & A.b & A.d & T.b & T.be & T.g & T.s & G.b & G.c & P.c & P.n & & \\
\hline \multicolumn{14}{|l|}{ Anacardiaceae } \\
\hline $\mathbf{\square}^{*}$ Anacardium occidentale L. & B & & + & & & & & & & + & & 2 & ps \\
\hline - $\downarrow$ Lannea acida A. Rch. s.l. & A & & & & & + & + & & & & & 2 & ps \\
\hline - $\downarrow$ Lannea barteri (Oliv.) Engl. & A & & & & & + & & & & & & 1 & ps \\
\hline •Spondias mombin L. & A & & & + & & & & + & & + & & 3 & $\mathrm{~s}$ \\
\hline \multicolumn{14}{|l|}{ Annonaceae } \\
\hline -*Annona senegalensis Pers. & $\mathrm{B}$ & & & & & & & & & + & & 1 & ps \\
\hline \multicolumn{14}{|l|}{ Apocynaceae } \\
\hline - $\downarrow$ Holarrhena floribunda (G. Don) Dur. \& Schinz. & A & & & & & & & & & + & & 1 & ps \\
\hline Thevetia peruviana (Pers.) K. Schum. & $\mathrm{a}$ & & & & + & + & & & & & & 2 & ps \\
\hline \multicolumn{14}{|l|}{ Asclepiadaceae } \\
\hline -Calotropis procera (Aiton) W. T. Aiton & $\mathrm{S}$ & & & & & + & & & & + & & 2 & ps \\
\hline \multicolumn{14}{|l|}{ Bignoniaceae } \\
\hline Crescentia cujete L. & $\mathrm{a}$ & & & + & & & & + & & & & 2 & ps \\
\hline -Newbouldia laevis (P. Beauv) Seemann ex Bureau & A & & & + & & & + & + & & & & 3 & s \\
\hline -Spathodea campanulata P. Beauv. & A & & & & + & & & & & & & 1 & ps \\
\hline \multicolumn{14}{|l|}{ Bombacaceae } \\
\hline -*Adansonia digitata $\mathrm{L}$. & A & & & + & & + & & + & & & & 3 & $\mathrm{~s}$ \\
\hline & & & 1677 & & & & & & & & & & \\
\hline
\end{tabular}


J G. HOUÉNON et al. / Int. J. Biol. Chem. Sci. 6(4): 1669-1686, 2012

$\mathbf{0}$ Bombax costatum Pellegr. \& Vuillet

-Ceiba pentandra (L.) Gaertn.

D. Rhodognaphalon brevicuspe (Sprague) Roberty

A

A

Boraginaceae

Cordia myxa L.

$+\frac{10}{20}$

$++$

$+$

Casuarinaceae

- Casuarina equisetifolia L.

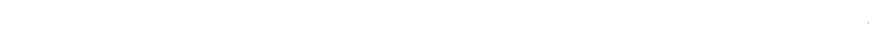

Celtidaceae

Trema orientalis (L.) Blume

A

$+$

$+\quad+$

$4 \quad 5$

Combretaceae

- $\downarrow$ Anogeissus leiocarpa (DC.) Guill. \& Perr.

$\mathbf{0}$ Combretum nigricans Lepr. ex. Guill. \& Perr.

Laguncularia racemosa (L.) Gaertn.f.

$\checkmark$ Terminalia catappa L.

- $\$$ Terminalia glaucescens Planch. ex Benth.

$\checkmark$ Terminalia mantaly H. Perrier

A

$+$

$+$

2

ps

\section{Euphorbiaceae}

-Aleurites moluccana (L.) Willd.

Antidesma venosum E. Mey. ex Tul.

-Bridelia ferruginea Benth.

-Croton gratissimus Burch.

Hevea brasiliensis (Willd. ex Juss.) Müll. Arg.

\begin{tabular}{|c|c|c|c|c|c|c|c|}
\hline A & + & & & & & 1 & ps \\
\hline A & + & & & & & 1 & ps \\
\hline $\mathrm{a}$ & & & & & & 1 & ps \\
\hline A & & + & + & + & + & 4 & $\mathrm{~S}$ \\
\hline A & + & & & & & 1 & ps \\
\hline A & & + & + & + & + & 4 & $\mathrm{~s}$ \\
\hline
\end{tabular}

A

a

a

A

A

$+$

$+$

ps 
J G. HOUÉNON et al. / Int. J. Biol. Chem. Sci. 6(4): 1669-1686, 2012

-Hymenocardia acida Tul.

-Jatropha curcas L.

Jatropha multifida L.

*Manihot esculenta Crantz.

Uapaca togoensis Pax

\begin{tabular}{|c|c|c|c|c|}
\hline $\mathrm{a}$ & + & + & & 2 \\
\hline $\mathrm{a}$ & + & + & & 2 \\
\hline $\mathrm{a}$ & & & + & 1 \\
\hline s & + & + & & 2 \\
\hline A & & & + & 1 \\
\hline
\end{tabular}

Irvingiaceae

*Irvingia gabonensis (Aubry-Lecomte ex. O’Rorke)

Baill.

A

$+$

Lauraceae

- Persea americana Mill.

A

$4 \quad s$

Leguminosae-Caesalpinioideae

$\mathbf{0}$ Afzelia africana $\mathrm{Sm}$.

- $\downarrow$ Daniellia oliveri (Rolfe) Hutch. et Dalziel

- *Detarium microcarpum Guill. \& Perr.

-Isoberlinia doka Craib \& Stapf

-Piliostigma reticulatum (DC.) Hochst.

-\$Senna siamea (Lam.) H. S. Irwin \& Barneby

Senna surattensis (Burm.f.) H. S. Irwin \& Barneby

- $*$ *Tamarindus indica L.

A

$+$

\section{Leguminosae-Mimosoideae}

$\checkmark$ Acacia auriculiformis A. Cunn. ex. Benth.

$\checkmark$ Acacia mangium Willd.

Albizia adianthifolia (Schumach.) F. Wight

\begin{tabular}{|c|c|c|c|c|c|c|c|c|}
\hline $\mathrm{A}$ & & & & & + & + & 2 & ps \\
\hline A & & & & + & + & & 2 & ps \\
\hline A & + & & & & & & 1 & ps \\
\hline A & & & & + & & & 1 & ps \\
\hline $\mathrm{a}$ & + & & & + & + & & 3 & $\mathrm{~s}$ \\
\hline A & & + & + & + & + & + & 5 & fs \\
\hline $\mathrm{a}$ & & & + & & & & 1 & ps \\
\hline A & + & & & & & & 1 & ps \\
\hline
\end{tabular}

\section{A}

A

A
$+$ 
J G. HOUÉNON et al. / Int. J. Biol. Chem. Sci. 6(4): 1669-1686, 2012

\begin{tabular}{|c|c|c|c|c|c|c|c|c|c|c|c|c|}
\hline Albizia glaberrima (Schumach. \& Thonn.) Benth. & A & & & & & + & & + & & & 2 & ps \\
\hline Albizia lebbeck (L.) Benth. & A & + & + & & + & & & & & & 3 & $\mathrm{~s}$ \\
\hline Albizia zygia (DC.) J. F. Macbr. & A & & & + & & & & & & & 1 & ps \\
\hline -Dichrostachys cinerea (L.) Wight et Arn. & $\mathrm{a}$ & + & & & & & & & & & 1 & ps \\
\hline Leucaena leucocephala (Lam.) De Wit & A & & & & & & + & & + & & 2 & ps \\
\hline - *Parkia biglobosa (Jacq.) R. Br. ex Benth. & A & + & + & & + & & & & & & 3 & $\mathrm{~s}$ \\
\hline Pithecellobium dulce (Roxb.) Benth. & $\mathrm{a}$ & & + & & + & & & & & & 2 & ps \\
\hline$\checkmark \mathbf{a} *$ Prosopis africana (Guill. \& Perr.) Taub. & A & + & & & & & & & & & 1 & ps \\
\hline \multicolumn{13}{|l|}{ Leguminosae-Papilionoideae } \\
\hline Dalbergia ecastaphyllum (L.) Taub. & $\mathrm{a}$ & & & & + & & & & & & 1 & ps \\
\hline -Erythrina senegalensis DC. & $\mathrm{a}$ & & & & & & & & & & & \\
\hline Erythrina variegata $\mathrm{L}$. & $\mathrm{a}$ & & & & & & + & & + & & 2 & ps \\
\hline -Lonchocarpus sericeus (Poir.) Kunth & A & + & & & & & & & & & 1 & ps \\
\hline $\mathbf{D} \gg$ Pterocarpus erinaceus Poir. & A & + & & & & & + & & & & 2 & ps \\
\hline Pterocarpus santalinoides L'Her. ex DC. & A & & & & & & & & + & + & 2 & ps \\
\hline \multicolumn{13}{|l|}{ Loranthaceae } \\
\hline Agelanthus dodoneifolius (DC.) Polh. \& Wiens & $\mathrm{a}$ & & & & + & & & & & & 1 & ps \\
\hline Globimetula cupulata (DC.) Danser. & $\mathrm{a}$ & & & & & & & + & & & 1 & ps \\
\hline \multicolumn{13}{|l|}{ Malvaceae } \\
\hline Hibiscus rosa-sinensis $\mathrm{L}$. & $\mathrm{s}$ & & & & + & & & & & & 1 & ps \\
\hline \multicolumn{13}{|l|}{ Meliaceae } \\
\hline $\mathbf{0} \diamond$ Azadirachta indica A. Juss. & A & & + & & + & & + & & & & 3 & $\mathrm{~s}$ \\
\hline
\end{tabular}


J G. HOUÉNON et al. / Int. J. Biol. Chem. Sci. 6(4): 1669-1686, 2012

\begin{tabular}{|c|c|c|c|c|c|c|c|c|c|}
\hline \multirow{3}{*}{$\begin{array}{l}\text { - } \downarrow \text { Khaya senegalensis (Desr.) A. Juss. } \\
\mathbf{0} \text { Pseudocedrela Kotschyi (Schweinf.) Harms. } \\
\text { Trichilia prieureana A. Juss. }\end{array}$} & \multirow{3}{*}{$\begin{array}{l}\mathrm{A} \\
\mathrm{A} \\
\mathrm{A}\end{array}$} & & & \multirow[t]{3}{*}{+} & \multirow[t]{3}{*}{+} & & & \multirow{2}{*}{$\begin{array}{l}2 \\
1\end{array}$} & \multirow{2}{*}{$\begin{array}{l}\text { ps } \\
\text { ps }\end{array}$} \\
\hline & & + & & & & & & & \\
\hline & & & & & & + & & 1 & ps \\
\hline \multicolumn{10}{|l|}{ Moraceae } \\
\hline$\checkmark$ Antiaris toxicaria Lesch. & A & & & & & & + & 1 & ps \\
\hline *Artocarpus altilis (Parkinson) Fosberg. & A & & + & & & + & & 2 & ps \\
\hline Ficus ingens (Miq.) Miq. & A & & & & + & & & 1 & ps \\
\hline -Ficus lutea Vahl & A & & + & + & & + & & 3 & $\mathrm{~s}$ \\
\hline -Ficus polita Vahl & A & & + & & & + & & 2 & ps \\
\hline - Ficus sur Forssk. & A & + & + & & + & & & 3 & $\mathrm{~s}$ \\
\hline -Ficus umbellata Vahl & A & & & & & + & + & 2 & ps \\
\hline$\checkmark$ Milicia excelsa (Welw.) C. C. Berg & A & & + & & + & + & & 3 & $\mathrm{~s}$ \\
\hline \multicolumn{10}{|l|}{ Moringaceae } \\
\hline Moringa oleifera Lam. & A & & & & + & + & & 2 & ps \\
\hline \multicolumn{10}{|l|}{ Myrtaceae } \\
\hline$\checkmark$ Eucalyptus torrelliana F. Muell. & A & & + & & & & & 1 & ps \\
\hline - Psidium guajava $\mathrm{L}$. & $\mathrm{a}$ & & & & + & & + & 2 & ps \\
\hline \multicolumn{10}{|l|}{ Ochnaceae } \\
\hline -Lophira lanceolata Teigh. ex Keay. & A & & & & + & & & 1 & ps \\
\hline \multicolumn{10}{|l|}{ Rhizophoraceae } \\
\hline Cassipourea barteri (Hook. f.) N. E. Br. & $\mathrm{a}$ & & + & & & & & 1 & ps \\
\hline
\end{tabular}

Rubiaceae 
J G. HOUÉNON et al. / Int. J. Biol. Chem. Sci. 6(4): 1669-1686, 2012

-Crossopteryx febrifuga (G. Don.) Benth.

-Gardenia erubescens Stapf \& Hutch.

-Gardenia ternifolia Schumach. \& Thonn.

-Mitragyna inermis (Willd.) Kuntze

Morelia senegalensis A. Rich. ex DC.

-Morinda lucida Benth.

\section{Rutaceae}

- *itrus aurantifolia (Christm. \& Panzer) Swingle

- *itrus reticulata Blanco

- *itrus sinensis Osbeck

-Zanthoxylum zanthoxyloides (Lam.) Zepernick et

Timler

Sapindaceae

口*Blighia sapida König

\begin{tabular}{|c|c|c|c|c|}
\hline A & + & + & 2 & ps \\
\hline $\mathrm{a}$ & & + & 1 & ps \\
\hline $\mathrm{a}$ & + & & 1 & ps \\
\hline A & & + & 1 & ps \\
\hline $\mathrm{a}$ & + & & 1 & ps \\
\hline $\mathrm{A}$ & + & + & 3 & $\mathrm{~s}$ \\
\hline
\end{tabular}

A

A

A

A

Sapotaceae

*Manilkara zapota (L.) P. Royen

- *Vitellaria paradoxa C. F. Gaertn.

$+$

$+$

Solanaceae

Capsicum annuum L.

A

A +

A

$+$

s +

$+$

\section{Sterculiaceae}

-Cola gigantea A.Chev.

Cola millenii K. Schum.

-Cola nitida (Vent.) Schott \& Endl.

A

A

A
$+$

$\begin{array}{lll}+ & \\ + & + & +\end{array}$

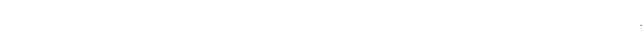

$\begin{array}{ccccc} & & + & 2 & \mathrm{ps} \\ + & + & + & 5 & \mathrm{fs} \\ + & + & 7 & \mathrm{tfs} \\ & & & & \\ + & & 1 & \mathrm{ps}\end{array}$

$+$

$+$

$3 \mathrm{~s}$

ps


J G. HOUÉNON et al. / Int. J. Biol. Chem. Sci. 6(4): 1669-1686, 2012

-Sterculia setigera Delile

*Theobroma cacao L.

$\checkmark$ Triplochiton scleroxylon K. Schum.

A

A

A
$+$

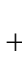

\section{Tiliaceae}

Glyphaea brevis (Spreng.) Monachino

1

\section{Verbenaceae}

$\checkmark$ Gmelina arborea Roxb.

$\checkmark$ Tectona grandis L.f.

-*Vitex doniana Sweet.

A

A

A

A

Nombre d'hôtes infestés par parasite

(1)

ra

TM : type morphologique des espèces hôtes (A : arbre, a : arbuste, S : suffrutex) ; Espèces de parasite (A.b: Agelantus brunneus, A.d: Agelantus dodoneifolius, T.b : Tapinanthus bangwensis, T.be : Tapinanthus belvisii, T.g : Tapinanthus globiferus, T.s: Tapinanthus sessifolius, G.b : Globimetula braunii, G.c : Globimetula cupulata, P.c : Phragmanthera capitata, P.n : Phragmanthera nigritana); + : présence du parasite sur l'hôte; Np : nombre d'espèces parasites par hôte; Sp : sensibilité parasitaire de l'hôte (hôte peu sensible (ps): 1 à 2 parasites, hôte sensible (s): 3 à 4 parasites, hôte fortement sensible (fs) : 5 à 6 parasites, hôte très fortement sensible (tfs) : 7 parasites et plus) ; $\mathbf{a}:$ médicinale ; * alimentaire ; : bois d'œuvre et de service. 


\section{DISCUSSION}

Les Loranthaceae des zones guinéenne et soudano-guinéenne du Bénin renferment 4 genres (Agelanthus, Globimetula, Phragmanthera et Tapinanthus) sur les 7 (Agelanthus, Berhautia, Englerina, Phragmanthera, Globimetula, Helixanthera et Tapinanthus) que compte la sous-région ouest africaine (Pohlill et Wiens, 1998), soit 57,1\%. Tous les genres décrits dans la Flore analytique du Bénin (Akoègninou et al., 2006) sont représentés.

Des 11 espèces mentionnées dans la Flore Analytique du Bénin (Akoègninou et al., 2006), 8, soit $72,7 \%$ sont aussi récoltées dans la présente étude. Mais Globimetula cupulata et Tapinanthus belvisii n'y figurent pas, elles sont récoltées pour la première fois au Bénin et sont donc nouvelles pour la Flore. Toutes les espèces sont rencontrées dans la zone guinéenne du Bénin. Par contre, 6 espèces sont communes aux zones guinéennes et soudano-guinéenne et aucune espèce n'est spécifique à la zone soudano-guinéenne. Ces résultats confirment les travaux de Akoègninou et al. (2006).

Cette diversité taxonomique de 4 genres et 10 espèces est largement supérieure à celle des 3 genres et 5 espèces obtenue au Mali (Boudet et Lebrun, 1986) et à celle de 3 genres et 6 espèces enregistrée par Boussim (1991, 2002) au Burkina Faso. Par contre, elle est nettement inférieure aux 6 genres et 19 espèces recensés en Côte d'Ivoire (Balle et Halle, 1961 ; Aké Assi, 1984) puis aux 6 genres et 25 espèces dénombrés au Cameroun (Balle, 1982). Les différences obtenues seraient non seulement liées à l'étendue des zones explorées mais aussi aux zones climatiques. En effet, l'analyse des données obtenues par les auteurs ci-dessus indique que la diversité taxonomique des Loranthaceae, en genres et en espèces, décroit avec l'aridité des zones; or la zone d'étude appartient au couloir de sécheresse Togo-Bénin dénommé Dahomey-Gap.

Les 105 plantes hôtes recensées appartiennent à 33 différentes familles botaniques. Ces résultats corroborent ceux de
Wiens (1998) qui a avait déjà signalé que les Loranthaceae sont des polyphages qui parasitent différentes familles botaniques n'ayant aucun lien phylogénétique. Boussim (1991), au Burkina Faso, a fait les mêmes observations sur le caractère polyphage du genre Tapinanthus. De plus, l'auteur a signalé l'épiparasitisme ou interparasitisme chez les Loranthaceae et c'est le cas de Tapinanthus globiferus qui parasite Agelanthus dodoneifolius.

La famille la plus diversifiée en terme d'espèces est la famille des Leguminosae avec un taux de $23,8 \%$, suivie des Euphorbiaceae $(8,6 \%)$, des Moraceae (7,6\%), Rubiaceae, Combretaceae et Sterculiaceae $(5,7 \%)$. Cette prédominance des Leguminosae n'est pas une particularité pour les hôtes de Loranthaceae mais une constance de la flore du Bénin. En effet, selon (Adomou et al., 2011), les 6 familles les plus diversifiées au Bénin sont les Leguminosae (14,8\%), Poaceae (9,3\%), Rubiaceae et Cyperaceae (5\% chacune), Asteraceae (4,6\%) et Euphorbiaceae (4,3\%). Les autres familles les plus diversifées en plantes-hôtes ne correspondent pas à celles de la flore du Bénin. En effet, les trois familles importantes du Bénin (Poaceae, Cyperacaeae et Asteracae) sont constituées surtout d'herbacées.

La diversité des plantes-hôtes est prédominée par les espèces médicinales avec un taux de $49,5 \%$ dont $37,1 \%$ pour les espèces exclusivement médicinales et $16,2 \%$ pour les espèces médicinales et alimentaires (Adjanohoun et al., 1989 ; Akoègninou et al., 2006). Ceci contribue à élargir la diversité des plantes médicinales car ces dernières sont toujours utilisées en rapport avec les planteshôtes pour guérir les maladies (Burkill, 1995).

\section{Conclusion}

Les Loranthaceae des zones guinéenne et soudano-guinéenne du Bénin comprennent 4 genres (Agelanthus, Globimetula, Phragmanthera et Tapinanthus) et 10 espèces dont 2 nouvelles (Globimetula cupulata et Tapinanthus belvisii) pour la flore du Bénin. Quatre de ces espèces sont rencontrées 
exclusivement dans la zone guinéenne et 6 sont communes aux deux zones phytogéographiques.

Les Loranthaceae ainsi recensées parasitent 105 espèces végétales dont 2 sont elles-mêmes des parasites. La sensibilité parasitaire de ces espèces varie d'une espèce à l'autre. Citrus sinensis a été identifiée comme l'espèce ayant la plus forte sensibilité parasitaire.

La forte sensibilité parasitaire de $C$. sinensis face à son importante valeur économique pose la problématique de l'impact de l'infestation des plantations d'agrumes par les Loranthaceae.

\section{RÉFÉRENCES}

Akoègninou A. 2004. Recherches Botaniques et Ecologiques sur les forêts actuelles du Bénin. Thèse d'Etat. Univ. de Cocody, Côte d'Ivoire, $326 \mathrm{p}$.

Akoègninou $\mathrm{A}$, van der Burg $\mathrm{WJ}$, van der Maesen LJG. 2006. Flore Analytique du Bénin. Backhuys Publishers: Leiden, Pays-Bas.

Anonyme. 2003. Troisième Recensement Général de la Population et de l'Habitat. Direction des Etudes Démographiques. INSAE-UNICEF-UNFPA-DDC.

Arbonnier M. 2000. Arbres, Arbustes et Lianes des Zones Sèches d'Afrique de l'Ouest. CIRAD, MNHN, UICN.

Aubréville A. 1963. Classification des formes biologiques des plantes vasculaires en milieu tropical. Adansonia, 111(2): 221225.

Balle S, Hallé N. 1962. Les Loranthaceae de la Côte d'Ivoire. Adansonia, 1: 208-265.

Balle S. 1982. Flore du Cameroun. Les Loranthaceae, Stabié, Leroy JF (éds). DGRST éd. DGRST: Yaoundé ; 23p.

Boudet G, Lebrun JP. 1986. Catalogue des plantes vasculaires du Mali. Etudes et synthèses, IEMTV.

Boussim IJ. 1991. Contribution à l'étude des Tapinanthus parasites du karité au Burkina-Faso. Thèse à de $3^{\mathrm{e}}$ cycle à l'Université de Ougadougou, BurkinaFaso, 130 p.
Barlow BA. 1983. Biogeography of Loranthaceae and Viscaceae. In The Biology of Mistletoes, Calder M, Bernhardt P (eds). Academic Press: Sydney; 19-46.

Chevalier A. 1946. L'arbre à beurre d'Afrique et l'avenir de sa culture. Oléagineux, 1: 711.

Dibong SD, Din N, Priso RJ, Taffouo VD, Fankem H, Salle G, Amougou A. 2008. Parasitism of host trees by the Loranthaceae in the region of Douala (Cameroon). African Journal of Environnemental Science and Technology, 2(11): 371- 378.

Edouard JA. 1989. Les phanérogames parasites de la Martinique. Ecologie et quelques aspects de leur biologie. Thèse de Doctorat de l'Université Pierre et Marie Curie, Paris, 305 p.

Fineran BA, Hocking PJ. 1983. Features of parasitism, Morphology and haustorial Anatomy in Loranthaceous Root Parasites. In The Biology of Mistletoes, Calder M, Bernhardt P (eds). Academic Press: Sydney; 205-258.

Hocking PJ, Fineran BA. 1983. Apects of the nutrition of root parasitic Loranthaceae. In The Biology of Mistletoes, Calder M, Bernhardt P (eds). Academic Press: Sydney; 229-258.

Hoffmann G. 1994. Contribution à l'étude des phanérogames parasites du Burkina Faso et du Mali. Quelques aspects de leur Ecologie, Biologie et Techniques de lutte. Thèse de Doctorat en Sciences de l'Univ. de Droit, d'Economie et des Sciences d'Aix-Marseille, 177p.

Hutchinson J, Dalziel JM. 1954-1972. Flora of West Tropical Africa (Vol.1 et 2). Whitefriars: London.

Lebrun JP, Stork AL. 1991-1997. Enumération des Plantes à Fleurs d'Afrique Tropicale ( $\mathrm{Vol}$ 1, 2, 3). Conservatoire et Jardin Botanique de la ville de Genève.

Loubreau-Callen D, Damblon F. 1994. Spectre pollinique des miels de l'abeille Apis mellifera Lin. (Hymenoptera, 
Apidae) et zones de végétation en Afrique occidentale tropicale et méditerranéenne. Grana, 33: 245-253.

Mallmaire A. 1945. Notes de phytopathologie soudanaise. Compt. Rend. Congrès Africaniste, Dakar, Sénégal, 518-528.

Natabou DF. 1987. Des plantes médicinales de la République Populaire du Bénin, p. 219.

Parker C, Riches CR. 1993. Parasittic Weeds of the World. CAB International: Wallingford. Oxon Ox 108 De. UK.

Polhill RM, Wiens D. 1998. Misteletoes of Africa. Royal Botanic Gardens: Kew.

Room PM. 1973. Ecology of the Mistletoe Tapinanthus bangwensis growing on cocoa in Ghana. Journal of Ecology, 61: 729-742.

Ruysen B. 1957. Le karité au Soudan. Agron. Trop., 12(2): 144-172.

Traoré D, Da Kouhété P, Bamba M. 1995. Lutte contre les plantes vasculaires parasites du karité et du néré, dans le Nord de la Côte d'Ivoire - Cas des Départements de Korhogo, Boundiali, Ferkessèdougou et Tengréla, 71p.

Wiens D, Tolken HR. 1979. Loranthaceae. In Flora of Southern Africa, Leistner OA (ed). Botanical Research Institute: Pretoria. 